Márcia Astrês Fernandes ${ }^{\mathrm{a}}$

(iD) https://orcid.org/0000-0001-9781-0752

Daniel de Macêdo Rocha ${ }^{\mathrm{a}}$

iD https://orcid.org/0000-0003-1709-2143

Hellany Karolliny Pinho Ribeiro

(iD) https://orcid.org/0000-0002-3157-7924

Carliane da Conceição Machado Sousa ${ }^{a}$

(iD) https://orcid.org/0000-0002-6766-0500

aUniversidade Federal do Piauí (UFPI), Campus Ministro Petrônio Portela,

Enfermagem. Teresina, PI, Brasil.

Contato:

Márcia Astrês Fernandes

E-mail:

m.astres@ufpi.edu.br

Os autores declaram que o estudo não foi subvencionado e que não há conflitos de interesses.

Os autores informam que o trabalho não foi apresentado em evento científico.

\section{Riscos ocupacionais e intervenções que promovem segurança para a equipe de enfermagem oncológica}

\author{
Occupational risks and interventions to \\ promote safety for oncology nurses
}

\title{
Resumo
}

Objetivo: analisar as evidências científicas relacionadas aos riscos ocupacionais e às intervenções que promovem segurança no trabalho para a equipe de enfermagem oncológica. Métodos: revisão de literatura realizada nas bases de dados MEDLINE, Web of Science, Scopus, LILACS, IBECS e BDENF. Resultados: a busca identificou 17 estudos primários, publicados em inglês, no período de 2008 a 2017. Dentre os fatores que configuram riscos ocupacionais, destacam-se: o déficit no conhecimento; a pouca disponibilidade de equipamentos de proteção individual para manuseio dos medicamentos antineoplásicos; o estresse; e a ansiedade. Quanto às intervenções que promovem a segurança, evidenciam-se: a manutenção do ambiente laboral favorável ao desempenho da assistência; a disponibilidade de insumos; o bom relacionamento interpessoal; a organização do trabalho; o dimensionamento profissional adequado; o conhecimento, a disponibilidade e o incentivo ao uso dos equipamentos de proteção; e a compreensão dos riscos ocupacionais aos quais os profissionais estão expostos. Conclusão: o estudo possibilitou a identificação de fatores associados à atividade laboral que comprometem a saúde da equipe de enfermagem oncológica, demonstrando a necessidade de intervenções voltadas para a melhoria das relações interprofissionais, a capacitação dos profissionais e o oferecimento de um ambiente de trabalho seguro e condições organizacionais que promovam a saúde dos trabalhadores.

Palavras-chave: equipe de enfermagem; enfermagem oncológica; riscos ocupacionais; saúde do trabalhador.

\begin{abstract}
Objective: to analyze scientific evidence on occupational risks and interventions aimed at promoting workplace safety for oncology nurses. Methods: literature review carried out in MEDLINE, Web of Science, Scopus, LILACS, IBECS, and BDENF databases. Results: the search returned 17 primary studies, published in English from 2008 to 2017. The most common factors identified as associated with occupational risks were knowledge deficit, insuficient personal protective equipment (PPE) available for handling anticancer drugs, stress, and anxiety. The interventions identified as promoting occupational safety were: keeping the work environment suitable for care performance; supplies availability; good interpersonal relationship; work organization; suitable professional dimensioning; availability, awareness and encouragement on the use of PPE; and the understanding of the occupational risks to which workers are exposed. Conclusion: the results enable the identification of work-related factors that endanger the oncology nursing teams' health, evincing the need for interventions aimed at improving interprofessional relationships, qualifying professionals, and offering a safe working environment and organizational conditions that will promote workers' health.
\end{abstract}

Keywords: nursing team; oncological nursing; occupational risks; occupational health. 


\section{Introdução}

O ambiente ocupacional é marcado por riscos que resultam na vulnerabilidade dos profissionais de saúde, dentre eles os que compõem a equipe de enfermagem, constituindo uma das categorias mais expostas a eventos estressores e desgastantes com potencial para determinar processos de adoecimento ${ }^{1}$. Isso decorre das peculiaridades do trabalho, bem como de sua organização e divisão, pois os profissionais de enfermagem permanecem expostos a esse ambiente durante toda a jornada laboral e por grande parte da vida produtiva ${ }^{2}$.

A configuração do perfil de adoecimento dos trabalhadores de enfermagem tem sido influenciada, particularmente, pelas alterações no processo de trabalho determinadas pelas políticas econômicas e trabalhistas adotadas. Observa-se uma elevada carga horária de trabalho, pouca autonomia e baixa remuneração, relações hierárquicas rigidamente demarcadas e elevadas exigências com o propósito de alcançar os objetivos institucionais e melhorar os indicadores de produtividade e qualidade ${ }^{3}$.

Desenvolver a assistência de enfermagem, em especial no setor de oncologia, implica a exposição dos trabalhadores aos riscos intrínsecos desse processo de trabalho, podendo desencadear acidentes e doenças ocupacionais que repercutem na vida e na saúde do trabalhador ${ }^{4}$. Além disso, essa atividade apresenta complexidade e exigências que podem gerar estresse ocupacional, tendo em vista as características dessa especialidade que incluem tratamento prolongado e agressivo dos pacientes, efeitos colaterais, cirurgias mutilantes, sofrimento, medo, desespero e morte ${ }^{5}$.

Para lidar com essas condições, o planejamento e a implementação de intervenções laborais são fundamentais e requerem a avaliação dos processos de trabalho e dos riscos ocupacionais, assim como a definição dos papéis assumidos nos diferentes contextos assistenciais. Nessa perspectiva, são necessárias reflexões sobre o trabalho de enfermagem, visando minimizar os riscos que ameaçam as diferentes dimensões que compõem a saúde e a qualidade de vida desses trabalhadores ${ }^{1}$.

Em Saúde e Segurança Ocupacional, é indispensável realizar intervenções favoráveis aos trabalhadores, a fim de preservar a vida, a saúde e, consequentemente, a capacidade de trabalho dos enfermeiros, visto que uma equipe saudável evita o absentismo e reduz a necessidade de substituição de profissionais ${ }^{6}$. Nesse sentido, investigar as medidas de enfrentamento às condições adversas e os níveis de estresse da equipe de enfermagem é o primeiro estágio para propiciar ambientes de trabalho com gerenciamento de fatores estressantes ${ }^{7}$.

Considerando as especificidades e a alta complexidade assistencial, gerencial e organizacional da área de oncologia, é de grande importância a realização de estudos com a finalidade de aprofundar o conhecimento sobre os riscos ocupacionais e as ações que promovem a saúde dos profissionais inseridos nessa proposta de cuidado, uma vez que fornecem subsídios para a promoção da saúde do trabalhador, por meio da elaboração de estratégias positivas, coletivas e individuais ${ }^{8}$.

Diante do exposto, este estudo tem como objetivo analisar as evidências científicas relacionadas aos riscos ocupacionais e às intervenções que promovam segurança no trabalho para a equipe de enfermagem oncológica.

\section{Métodos}

Desenvolveu-se uma revisão de literatura para identificar, avaliar e sintetizar a qualidade de evidências encontradas ${ }^{9}$. Para tanto, esta investigação foi conduzida em seis etapas: formulação da questão de pesquisa; busca na literatura e seleção dos trabalhos; coleta de dados; avaliação crítica dos estudos escolhidos; interpretação dos resultados; e apresentação da revisão ou síntese do conhecimento.

A questão da pesquisa foi formulada por meio da estratégia PICO, em que foram considerados, como população, a equipe de enfermagem, como fenômeno de interesse, os riscos ocupacionais e, como contexto, a oncologia ${ }^{10}$. Dessa forma, a questão elaborada foi: quais são as evidências científicas dos riscos ocupacionais e das intervenções de prevenção relacionadas à atividade de trabalho desenvolvida pela equipe de enfermagem oncológica?

Realizou-se a busca na literatura em fevereiro de 2018 mediante consulta nas seguintes bases eletrônicas de dados: Medical Literature Analysis and Retrieval System Online (MEDLINE via PubMed), Web of Science (WoS), Scopus, Literatura LatinoAmericana e do Caribe em Ciências da Saúde (LILACS), Índice Bibliográfico Español en Ciencias de la Salud (IBECS) e Base de Dados da Enfermagem (BDENF) via Biblioteca Virtual em Saúde.

Para inclusão na pesquisa, foram considerados estudos de fontes primárias, publicados em inglês, português ou espanhol, no período de 2008 a 2017, que abordassem os riscos ocupacionais a que a equipe de enfermagem oncológica está exposta. Excluíram-se os artigos que se encontravam duplicados nas bases de dados, teses, dissertações, editoriais e revisões de literatura.

Para operacionalização da busca e recuperação dos estudos, empregaram-se descritores controlados (que são utilizados para a indexação de artigos nas bases de dados) e não controlados (palavras textuais e seus sinônimos, variações de grafia, siglas e correlatos) selecionados após consulta aos termos frequentes nos Descritores em Ciências da Saúde 
(DeCS) e Medical Subject Headings (MeSH) e nos títulos Cumulative Index to Nursing and Allied Health Literature (CINAHL) (Quadro 1).

A estratégia de busca foi gerada mediante a combinação dos descritores com os operadores booleanos AND e OR, sendo adaptada de acordo com o acrônimo PICO e as especificidades para o acesso a cada base. O Quadro 2 apresenta a expressão de busca realizada na MEDLINE, adaptada para as demais bases analisadas.

As produções foram acessadas por meio do portal de periódicos da Coordenação de Aperfeiçoamento de Pessoal de Nível Superior (CAPES), sendo busca, seleção e inclusão realizadas por dois revisores de forma independente que, após a leitura de títulos e resumos, obtiveram índice de concordância superior a $80 \%$. Em caso de discordância, um terceiro revisor foi solicitado a emitir parecer sobre a inclusão ou não do estudo.

A extração dos dados dos artigos selecionados foi realizada com auxílio de um formulário próprio contendo variáveis relacionadas à identificação dos estudos (autores, ano, idioma e periódico de publicação), objetivos, método (delineamento e amostra), principais desfechos, resultados e conclusões. Quanto à análise, utilizaram-se os métodos descritivos, em que foi realizada a construção de quadros conforme as variáveis de interesse para esta investigação. Ainda, as evidências foram classificadas quanto à similaridade semântica e apresentadas em duas categorias temáticas.

Quadro 1 Descritores controlados e não controlados utilizados para construção da estratégia de busca

\begin{tabular}{|c|c|l|}
\hline \multicolumn{3}{|c|}{ MeSH e títulos CINAHL } \\
\hline \multirow{4}{*}{ P } & DC & Nursing, Team; Nurse Practitioners; Nurses; Licensed Practical Nurses. \\
\cline { 2 - 3 } & DNC & Team Nursing, Practical Nurses. \\
\cline { 2 - 3 } & DNC & $\begin{array}{l}\text { Team Nursing; Nurse Practitioner; Practitioner, Nurse; Practitioners, Nurse; Nurse; Personnel, Nursing; Nursing } \\
\text { Personnel. }\end{array}$ \\
\hline \multirow{2}{*}{ I } & DC & Occupational Health. \\
\cline { 2 - 3 } CO & DNC & Health, Occupational; Safety, Occupational; Occupational Safety; Employee Health; Health, Employee. \\
\cline { 2 - 3 } & DC & Medical Oncology; Oncology Nursing. \\
\hline \multirow{3}{*}{ PNC } & $\begin{array}{l}\text { Oncology, Medical; Clinical Oncology; Oncology, Clinical; Oncologic Nursing; Cancer Nursing; Nursing, Cancer; } \\
\text { Oncological Nursing. }\end{array}$ \\
\hline & DC & Equipe de Enfermagem, Profissionais de Enfermagem, Enfermeiras e Enfermeiros, Técnicos de Enfermagem. \\
\hline \multirow{2}{*}{ I } & DNC & Enfermeira, Enfermeira e Enfermeiro, Enfermeiro e Enfermeira, Enfermeiros e Enfermeiras. \\
\cline { 2 - 3 } & DC & Saúde do Trabalhador. \\
\hline \multirow{2}{*}{ CO } & DNC & $\begin{array}{l}\text { Segurança no Trabalho, Saúde Ocupacional, Segurança do Trabalho, Segurança Ocupacional, Saúde dos Trabalhadores, } \\
\text { Segurança dos Trabalhadores. }\end{array}$ \\
\cline { 2 - 3 } & DC & Oncologia, Enfermagem Oncológica. \\
\hline
\end{tabular}

DC: descritor controlado; DNC: descritor não controlado.

Quadro 2 Expressão de busca gerada na base de dados MEDLINE via PubMed

\begin{tabular}{|c|c|}
\hline \multicolumn{2}{|r|}{ Expressão de busca } \\
\hline$P$ & $\begin{array}{l}\text { (“nursing, team”[Mesh]) OR “nurse practitioners”[Mesh] OR “nurses”[Mesh] OR “licensed practical nurses”[Mesh] OR "team } \\
\text { nursing” OR “nurse practitioner" OR “practitioner, nurse” OR “practitioners, nurse” OR “nurse” OR “personnel, nursing” OR } \\
\text { "nursing personnel” }\end{array}$ \\
\hline I & $\begin{array}{l}\text { (“occupational health"[Mesh]) OR "health, occupational") OR “safety, occupational”) OR “occupational safety") OR “employee } \\
\text { health") OR “health, employment") }\end{array}$ \\
\hline $\mathrm{CO}$ & $\begin{array}{l}\text { (“medical oncology”[Mesh]) OR “oncology nursing”[Mesh])) OR “oncology nursing”) OR “clinical oncology”) OR “oncology, } \\
\text { clinical”) OR “oncologic nursing”) OR “cancer nursing”) OR “nursing, cancer”) OR “oncological nursing”) }\end{array}$ \\
\hline \multicolumn{2}{|r|}{ (P) AND (I) AND (CO) } \\
\hline \multicolumn{2}{|r|}{ 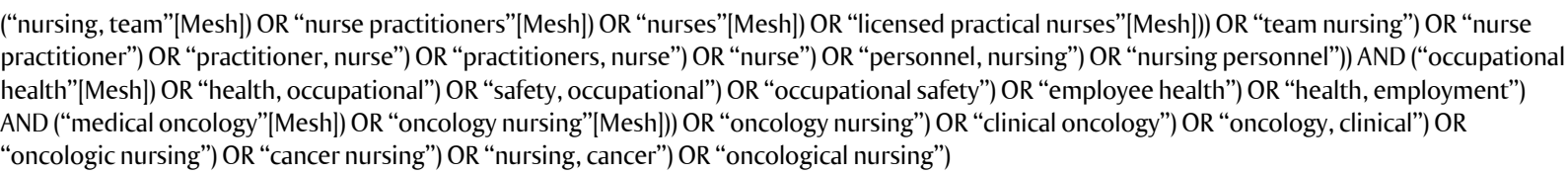 } \\
\hline
\end{tabular}




\section{Resultados}

A busca totalizou 112 artigos, entre os quais 26 foram excluídos por estarem duplicados nas bases, assim como por compreenderem teses, dissertações, revisões e editoriais, resultando em 98 estudos para leitura prévia de títulos. O conjunto final selecionado resultou em 17 publicações. A Figura 1 descreve o percurso realizado para identificação, inclusão e exclusão dos estudos, segundo a base consultada.

Entre os 17 estudos analisados, verificou-se que a maioria era em inglês e havia um predomínio de estudos quantitativos (13). Destacou-se o periódico Clinical Journal of Oncology Nursing com 3 produções. A maior parte dos artigos (6) foi publicada no ano de 2015. A síntese dos resultados foi dividida em duas categorias: riscos ocupacionais para saúde da equipe de enfermagem oncológica; e intervenções que promovem segurança para a equipe de enfermagem oncológica.

O Quadro 3 apresenta os estudos selecionados conforme autor principal, periódico, ano de publicação, delineamento, amostra (n), país do estudo, principais resultados e conclusões.

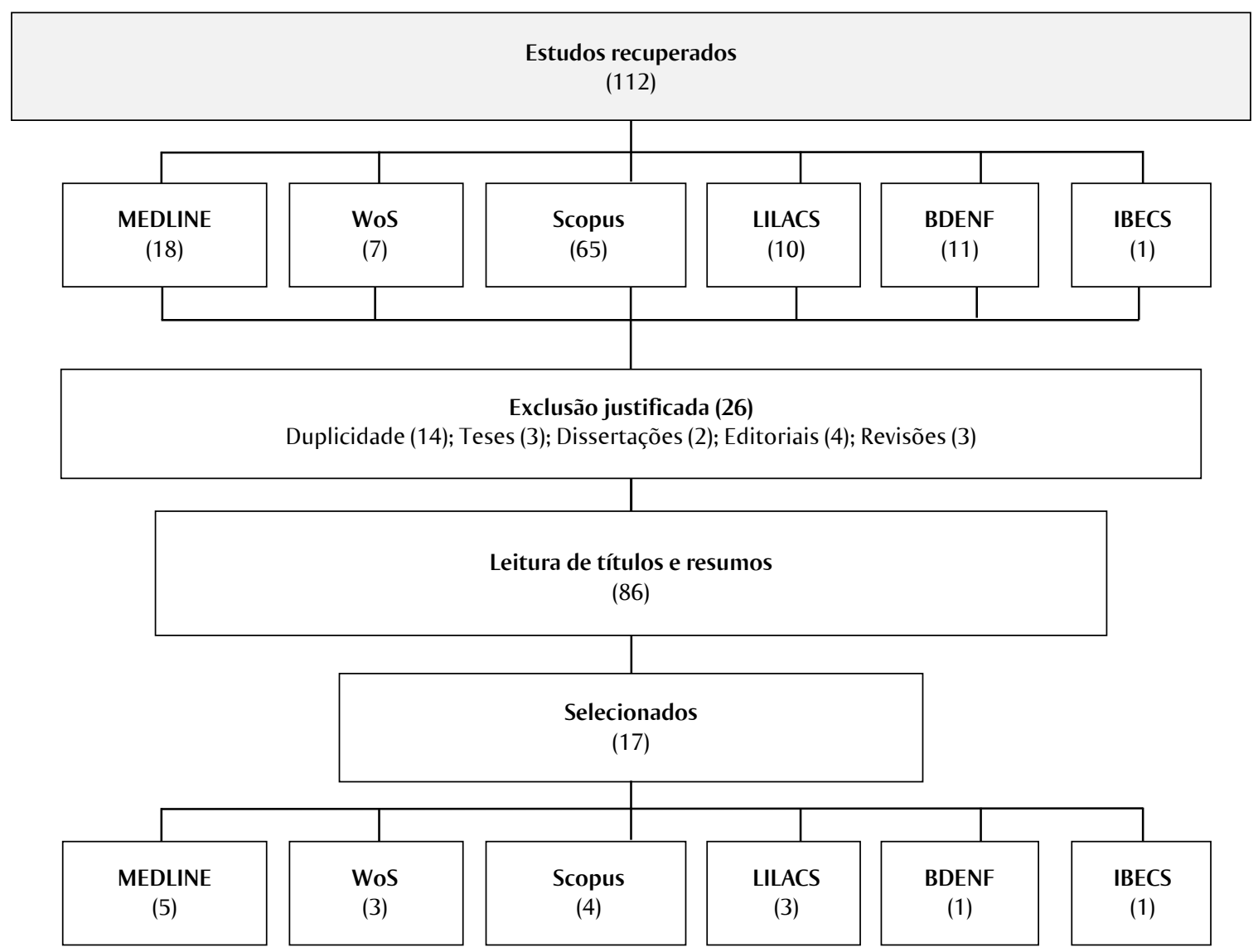

Figura 1 Percurso para identificação, inclusão e exclusão nas bases eletrônicas investigadas 
Quadro 3 Artigos selecionados $(n=17)$ segundo categoria temática, autor principal, periódico, ano de publicação, delineamento, tamanho da amostra, país do estudo e principais resultados

\begin{tabular}{|c|c|c|}
\hline \multicolumn{3}{|c|}{ Categoria 1 - Riscos ocupacionais para saúde da equipe de enfermagem oncológica } \\
\hline Autor principal, periódico e ano & $\begin{array}{l}\text { Delineamento, } \\
\text { amostra }(n) \text { e país }\end{array}$ & Principais resultados e conclusões \\
\hline $\begin{array}{l}\text { Crickman R. Clinical Journal of Oncology } \\
\text { Nursing, 2017 }\end{array}$ & $\begin{array}{l}\text { Estudo qualitativo, (31), } \\
\text { Estados Unidos da América }\end{array}$ & $\begin{array}{l}\text { Déficit no conhecimento dos enfermeiros sobre quimioterapia, pouca } \\
\text { disponibilidade de equipamento de proteção individual (EPI) para } \\
\text { manuseio de medicamentos. }\end{array}$ \\
\hline $\begin{array}{l}\text { De la Fuente-Solana El. European } \\
\text { Journal of Oncology Nursing, } 2017^{12}\end{array}$ & $\begin{array}{l}\text { Estudo quantitativo, (101), } \\
\text { Espanha }\end{array}$ & $\begin{array}{l}\text { Contato com pessoas diagnosticadas com doenças potencialmente } \\
\text { fatais, intenso sentimento de luto pela morte frequente de } \\
\text { pacientes, exaustão emocional, diminuição da realização pessoal } \\
\text { e despersonalização. O estudo mostra número significativo de } \\
\text { enfermeiras oncológicas com burnout grave. }\end{array}$ \\
\hline $\begin{array}{l}\text { Colvin CM. Clinical Journal of Oncology } \\
\text { Nursing, } 2016^{13} \text {. }\end{array}$ & $\begin{array}{l}\text { Estudo quantitativo, (33), } \\
\text { Estados Unidos da América }\end{array}$ & $\begin{array}{l}\text { Divergência com relação ao comportamento objetivo e subjetivo, } \\
\text { pois, embora nas autoavaliações os profissionais demonstrassem } \\
\text { conhecimento sobre o uso correto de EPI, na prática não o utilizavam } \\
\text { da forma recomendada. }\end{array}$ \\
\hline $\begin{array}{l}\text { Karanikola MNK. Revista da Escola de } \\
\text { Enfermagem da USP, 2016 } \\
\end{array}$ & $\begin{array}{l}\text { Estudo quantitativo, (72), } \\
\text { Grécia }\end{array}$ & $\begin{array}{l}\text { Ansiedade, contato frequente com a morte, baixa qualidade de } \\
\text { interação entre os profissionais, cargas de trabalho excessivas. }\end{array}$ \\
\hline Friese CR. Trials, $2015^{15}$. & $\begin{array}{l}\text { Estudo quantitativo, (382), } \\
\text { Estados Unidos da América }\end{array}$ & $\begin{array}{l}\text { Ausência de recursos materiais para proteção pessoal e de intervenções } \\
\text { voltadas para a melhoria da prática assistencial. }\end{array}$ \\
\hline $\begin{array}{l}\text { Davidson JE. The Journal of Nursing } \\
\text { Administration, } 2016^{16} \text {. }\end{array}$ & $\begin{array}{l}\text { Estudo quantitativo, (117), } \\
\text { Estados Unidos da América }\end{array}$ & $\begin{array}{l}\text { Desfechos negativos dos pacientes podem gerar culpa e angústia } \\
\text { moral nos profissionais de enfermagem, resultando em absentismo e } \\
\text { doenças psicológicas. O absentismo dos colegas de trabalho gera um } \\
\text { acúmulo de atividades. }\end{array}$ \\
\hline $\begin{array}{l}\text { Trindade LL. Revista de Pesquisa, } \\
\text { Cuidado é Fundamental, } 2015^{17} \text {. }\end{array}$ & Estudo qualitativo, (31), Brasil & $\begin{array}{l}\text { Quantitativo inadequado de profissionais, estrutura física precária e } \\
\text { falta de treinamento dos profissionais. }\end{array}$ \\
\hline $\begin{array}{l}\text { Poulsen AA. Journal of Medical Imaging } \\
\text { and Radiation Oncology, } 2015^{18} \text {. }\end{array}$ & $\begin{array}{l}\text { Estudo quantitativo, (70), } \\
\text { Austrália }\end{array}$ & $\begin{array}{l}\text { Elevado grau de estresse, insatisfação com o autocuidado e má } \\
\text { qualidade do sono. }\end{array}$ \\
\hline $\begin{array}{l}\text { Nascimento L. Revista de Enfermagem } \\
\text { UFPE On Line, } 2011^{19} \text {. }\end{array}$ & Estudo qualitativo, (6), Brasil & $\begin{array}{l}\text { O não uso de EPI, o uso incorreto na manipulação de substâncias } \\
\text { quimioterápicas e o desconhecimento dos riscos ocupacionais aos } \\
\text { quais os profissionais estão expostos. }\end{array}$ \\
\hline \multicolumn{3}{|c|}{ Categoria 2 - Intervenções que promovem segurança para equipe de enfermagem oncológica } \\
\hline $\begin{array}{l}\text { Ko W. Clinical Journal of Oncology } \\
\text { Nursing, } 2016^{20} \text {. }\end{array}$ & $\begin{array}{l}\text { Estudo quantitativo, (40), } \\
\text { Estados Unidos da América }\end{array}$ & $\begin{array}{l}\text { Estratégias utilizadas para o gerenciamento do estresse: recurso } \\
\text { espiritual, como idas à capela; disponibilidade da gestão na busca de } \\
\text { ideias ou planos de melhoria; reuniões, conversas e discussão sobre } \\
\text { luto e perda de pacientes. }\end{array}$ \\
\hline Chen HC, Cancer Nursing, $2016^{21}$. & $\begin{array}{l}\text { Estudo qualitativo, (57), } \\
\text { China }\end{array}$ & $\begin{array}{l}\text { A medida adotada pelo hospital foi a centralização do preparo da } \\
\text { quimioterapia na farmácia oncológica, que possui equipamento } \\
\text { moderno e adequado. }\end{array}$ \\
\hline $\begin{array}{l}\text { Jeong KW, Asian Pacific Journal of } \\
\text { Cancer Prevention, } 2015^{22} \text {. }\end{array}$ & $\begin{array}{l}\text { Estudo quantitativo, (236), } \\
\text { Coreia do Sul }\end{array}$ & $\begin{array}{l}\text { Educação continuada sobre a manipulação de medicamentos } \\
\text { anticancerígenos, desenvolvimento de uma relação positiva entre } \\
\text { o nível de consciência e o desempenho da enfermagem quanto } \\
\text { às regulamentações de segurança na preparação, administração, } \\
\text { manuseio geral e descarte dos anticancerígenos. }\end{array}$ \\
\hline Umann J, Rev Rene, $2013^{23}$. & $\begin{array}{l}\text { Estudo quantitativo, (18), } \\
\text { Brasil }\end{array}$ & $\begin{array}{l}\text { O controle foi o fator mais utilizado para o enfrentamento dos } \\
\text { estressores que existem no ambiente ocupacional, em que as ações são } \\
\text { voltadas para o problema, como a tentativa de modificar os fatores que } \\
\text { causaram a situação e o diálogo com os colegas envolvidos no problema. }\end{array}$ \\
\hline $\begin{array}{l}\text { Polovich M, Oncology Nursing Forum, } \\
2012^{24} \text {. }\end{array}$ & $\begin{array}{l}\text { Estudo quantitativo, (185), } \\
\text { Estados Unidos da América }\end{array}$ & $\begin{array}{l}\text { Condições adequadas de organização do trabalho, tais como o número } \\
\text { de pacientes atendidos por dia, disponibilidade de EPI e clima de } \\
\text { segurança do local de trabalho. }\end{array}$ \\
\hline $\begin{array}{l}\text { Polovich M, Oncology Nursing Forum, } \\
2011^{25} \text {. }\end{array}$ & $\begin{array}{l}\text { Estudo quantitativo, (330), } \\
\text { Estados Unidos da América }\end{array}$ & $\begin{array}{l}\text { Aumento do uso de EPI por profissionais de enfermagem durante o } \\
\text { preparo de drogas perigosas, com destaque para as luvas. Porém, o uso } \\
\text { de gorros ainda é baixo. }\end{array}$ \\
\hline $\begin{array}{l}\text { Baykal U, European Journal of Oncology } \\
\text { Nursing, } 2009^{26} \text {. }\end{array}$ & $\begin{array}{l}\text { Estudo quantitativo, (171), } \\
\text { Turquia }\end{array}$ & $\begin{array}{l}\text { Uma pequena parcela dos quimioterápicos é preparada em farmácia. } \\
\text { Poucas enfermeiras fizeram cursos de curta duração sobre efeitos } \\
\text { colaterais da quimioterapia, métodos de proteção, entre outros. Os } \\
\text { enfermeiros oncológicos estão empregados em conformidade parcial } \\
\text { com as leis trabalhistas nacionais. }\end{array}$ \\
\hline $\begin{array}{l}\text { Rodrigues AB, Revista Latino- } \\
\text { americana de Enfermagem, } 2008^{27} \text {. }\end{array}$ & $\begin{array}{l}\text { Estudo quantitativo, (77), } \\
\text { Brasil }\end{array}$ & $\begin{array}{l}\text { Uso de estratégias de coping a fim de resolver os problemas que } \\
\text { surgiram no ambiente de trabalho, sendo as mais utilizadas: reavaliação } \\
\text { positiva, resolução de problema e autocontrole. }\end{array}$ \\
\hline
\end{tabular}




\section{Discussão}

\section{Riscos ocupacionais para saúde da equipe de enfermagem oncológica}

De acordo com os estudos selecionados, entre os fatores que comprometem a saúde da equipe de enfermagem oncológica e geram riscos ocupacionais, destacam-se: a escassez e/ou a baixa adesão ao uso de EPIs, o déficit no conhecimento desses profissionais quanto aos riscos aos quais estão expostos, alta carga de trabalho, baixa interação interprofissional e ambiente de trabalho precário. O fornecimento dos EPIs apropriados e o incentivo ao seu uso são deveres dos empregadores, no entanto, os EPIs designados para o trabalho com quimioterapia nem sempre são fornecidos, o que representa riscos consideráveis, visto que na oncologia se trabalha com medicamentos perigosos, que apresentam um grau elevado de toxicidade, podendo gerar problemas de saúde para os trabalhadores ${ }^{25}$.

Um estudo realizado com pré e pós-testes de conhecimento dos enfermeiros sobre exposição à quimioterapia evidenciou a importância do uso adequado do EPI, incluindo sua padronização, além da identificação de medicamentos perigosos e da educação dos profissionais que lidam com as substâncias. Testes de limpeza de superfície onde os quimioterápicos são manipulados foram realizados para determinar a contaminação por drogas perigosas e foram encontradas amostras positivas para o 5 -fluorouracil, confirmando a dificuldade de manter um ambiente livre de contaminação e a necessidade essencial do uso de EPI pelos profissionais ${ }^{11}$.

Apesar disso, uma pesquisa que avaliou os efeitos dos antineoplásicos na saúde dos trabalhadores que os manipulam constatou que a administração frequente desses medicamentos, a baixa adesão e a existência de barreiras relacionadas ao uso de EPIs, assim como a percepção de baixo risco à saúde no trabalho, pelos enfermeiros oncológicos, são aspectos que deixam esses profissionais vulneráveis ${ }^{15}$.

$\mathrm{Na}$ atividade de atendimento oncológico, diversos fatores podem comprometer a saúde do profissional de enfermagem, como cansaço, fadiga, estresse, cefaleia recorrente, alterações de humor decorrente dos óbitos e de situações de emergência dos pacientes, além das condições de trabalho e dos problemas de relacionamento entre a equipe de enfermagem ${ }^{27}$. O exercício da enfermagem nas unidades de assistência oncológica é caracterizado por instabilidades, incertezas, imediatismo e necessidade de enfrentamento de situações emergenciais, que decorrem tanto das exigências quanto da diversidade de tarefas e especificidade das funções exercidas. Dessa forma, o que mais compromete a equipe de enfermagem nessa atividade é o desgaste emocional em consequência do trabalho, da falta de recursos humanos e materiais ${ }^{23}$. Estudos conduzidos na Espanha e na Grécia também descreveram esse fenômeno, corroborando o fato de que a exaustão emocional e a despersonalização profissional têm uma correlação positiva com ansiedade e depressão, resultando em um número significativo de enfermeiros oncológicos nos estágios mais graves de sofrimento por burnout ${ }^{12,14}$.

Além disso, enfermeiras que administram quimioterapia e outras drogas potencialmente perigosas à saúde dos que as manuseiam precisam conhecer os riscos da exposição ocupacional e da necessidade do uso dos recursos de proteção para desenvolver suas atividades. Estudos observaram déficit no conhecimento desses trabalhadores com relação aos riscos a que estão expostos na administração de antineoplásicos e quanto à importância do uso de EPI ${ }^{18,19}$.

O baixo nível de conhecimento das normas de segurança quanto à preparação dos medicamentos é um dos riscos identificados à saúde dos profissionais de enfermagem ${ }^{22}$. A manipulação de substâncias quimioterápicas pode comprometer a saúde do trabalhador, uma vez que, ao manusear drogas antineoplásicas, ele pode inalar, ingerir ou se expor pelo contato desses agentes com mucosas e pele, podendo ocasionar diversos tipos de problemas de saúde ${ }^{19}$.

$\mathrm{Na}$ Turquia, enfermeiros de oncologia que administravam drogas citotóxicas estavam sobrecarregados, não dispunham de tempo livre, não recebiam por horas extras trabalhadas e enfrentavam outras deficiências quanto à segurança no trabalho $^{27}$. Segundo os autores, as condições de trabalho atendiam apenas parcialmente às leis nacionais e ignoravam os padrões da Organização Internacional do Trabalho (OIT) para os profissionais de saúde. Outras questões, como problemas de comunicação com pacientes e seus familiares, cargas horárias excessivas, conflitos com seus colegas de trabalho, falta de suprimentos, falta de apoio de gerentes e baixos salários, também foram observadas no estudo ${ }^{26}$.

Um estudo de abordagem qualitativa, desenvolvido em uma Unidade de Internação Oncológica do estado de Santa Catarina, evidenciou que o contexto ocupacional é marcado por diferentes conflitos entre as necessidades e possibilidades de gratificação e a restrição de satisfazê-las dentro do ambiente de trabalho. Desse modo, o grau de insatisfação profissional ultrapassou o comprometimento com o colega de trabalho, impactando diretamente nos níveis de absentismo, nos indicadores de saúde do trabalhador e na qualidade do cuidado, impactando diretamente a segurança do paciente ${ }^{17}$. 
Ademais, o ambiente de trabalho pode ter um efeito negativo sobre a vida pessoal, social e profissional, e pode resultar em mudanças associadas à qualidade dos cuidados de enfermagem, bem como nos relacionamentos interprofissionais ${ }^{14}$. Fatores relacionados com o cuidado de pacientes com tratamentos complexos e diagnósticos terminais podem favorecer tratamento despersonalizado para evitar o sofrimento excessivo dos profissionais ${ }^{12}$ diante de estresse, falta de treinamento, tristeza e necessidade de comunicar más notícias a familiares.

\section{Intervenções que promovem segurança para equipe de enfermagem}

As evidências disponíveis na literatura sobre os fatores que promovem segurança para equipe de enfermagem oncológica são relativamente recentes. Entre elas, destacam-se as intervenções relacionadas à melhoria do ambiente laboral para o desempenho da assistência. Ou seja, um local de trabalho em que há disponibilidade de insumos, boa organização do trabalho e relacionamento interpessoal, dimensionamento profissional adequado, bem como a promoção do conhecimento, a disponibilidade e o incentivo ao uso de EPI e a compreensão dos riscos ocupacionais aos quais os profissionais estão expostos.

Na dimensão paliativa, foram evidenciadas ações de enfermagem baseadas no problema, dentre elas: a gestão participativa do cuidado; atividades para educação continuada ou permanente; e reuniões periódicas para expor as dificuldades e os desafios do contexto assistencial. Além disso, a realização de práticas e treinamentos para sanar as dúvidas individuais foram responsáveis por promover mudanças comportamentais e ambientais, e por constituir elemento minimizador de estresse ${ }^{28}$.

Em situações complexas, como aquelas que ocorrem em unidades de oncologia, uma maior empatia e boa comunicação, acompanhadas de bondade e preocupação com os pacientes, podem favorecer os sentimentos gratificantes e evitar o surgimento de ansiedade nos enfermeiros. Por outro lado, a exposição a eventos adversos e à morte tornam os profissionais conscientes da sua própria mortalidade, aumentando a ansiedade e a depressão e provocando a exaustão ${ }^{20}$.

Nessa perspectiva, considera-se que o ambiente ocupacional interfere no funcionamento e na qualidade dos serviços, requerendo a identificação e mensuração dos fatores favoráveis às boas práticas do enfermeiro, assim como a redução de riscos e eventos estressores ${ }^{21,29}$.

Um estudo sobre tomada de decisão e suas influências no ambiente do trabalho oncológico e uma pesquisa conduzida para determinar os problemas de condições de trabalho e a vida profissional de enfermeiros de oncologia na Turquia, ressaltaram, como facilitadores para um ambiente laboral benéfico à saúde, a autonomia, o suporte organizacional, o controle do ambiente com infraestrutura adequada e a disponibilidade de recursos para o cuidar seguro ${ }^{16,26}$.

Nessa perspectiva, é necessário favorecer o desempenho das atividades dos trabalhadores, protegendo e promovendo sua saúde. Assim, medidas de biossegurança são fundamentais e devem direcionar ações de segurança frente aos riscos biológicos, químicos e físicos impostos pelo ambiente de trabalho. Considerando isso, são imprescindíveis orientações, capacitações e esclarecimentos para maior adesão e utilização correta dos equipamentos de proteção, além de garantir manuseio, transporte e descarte seguro de material biológico ${ }^{30}$.

Uma investigação sobre as preocupações dos enfermeiros em relação ao uso de EPI e um ensaio controlado randomizado com enfermeiros habilitados para infusão de quimioterapia enfatizaram a capacitação e o treinamento, aliados ao feedback da prática assistencial segura, como forma de compreensão e maior adesão ao uso de EPIs ${ }^{15,21}$.

Vários dos estudos selecionados por esta pesquisa consideram importante o investimento no processo motivacional e educativo, assim como na promoção da saúde no ambiente de trabalho, por meio de treinamentos, cursos e palestras que visem reduzir a exposição aos riscos e prevenir o surgimento das doenças ocupacionais. Para melhor adesão, essas ações devem contar com a participação ativa e coletiva dos trabalhadores ${ }^{11,13,19,20,22,24}$.

A organização do trabalho e a comunicação interprofissional são ferramentas importantes para avaliar e identificar a necessidade de ajustes a fim de reduzir ou remediar possíveis situações de insatisfação e dificuldade, diminuindo o sofrimento psicológico dos trabalhadores da enfermagem de oncologia. Além disso, a satisfação no trabalho está relacionada ao nível de compromisso da equipe ${ }^{14,17,18}$.

A assistência de enfermagem oncológica demonstra uma necessidade maior de estratégias de controle e exercício mental, em comparação a outras áreas de saúde. Sendo assim, no manejo do estresse ocupacional, estratégias de coping podem ser válidas, efetivas e seguras, proporcionando tolerância e redução das demandas externas e internas. Assim, é necessária a avaliação dessas estratégias, visando estabelecer os aspectos favoráveis à manutenção da saúde e prevenção do adoecimento ${ }^{13,24,28}$.

Várias das proposições para a promoção da segurança focam basicamente na educação ou capacitação dos enfermeiros sobre os riscos aos quais estão 
expostos, o que é necessário, mas insuficiente para proteger adequadamente os trabalhadores. Ao fazer isso, os estudos apresentam uma visão limitada sobre estratégias para lidar com a questão da saúde do trabalhador, ao invés de buscar ações mais efetivas para a proteção e promoção de sua saúde e segurança, como medidas relacionadas às condições e à organização do trabalho.

\section{Conclusão}

Este estudo identificou os diferentes aspectos ocupacionais que podem comprometer a saúde da equipe de enfermagem oncológica, aumentando a exposição a riscos e elevando as cargas físicas e psíquicas. Assim, situações relacionadas à rotina diária, conflitos, dilemas morais e éticos representaram fatores associados e determinantes dos níveis de segurança do profissional de enfermagem. A sobrecarga de trabalho, a insuficiência de recursos humanos e materiais de serviço, o sentimento de impotência ou a inabilidade em prover atendimento de qualidade e o pouco conhecimento relacionado aos riscos ocupacionais também configuraram condições de riscos.
Dessa forma, as evidências científicas encontradas elencaram como riscos ocupacionais aqueles associados à escassez e/ou baixa adesão ao uso de EPIs, ao déficit de conhecimento desses profissionais quanto aos riscos, à alta carga de trabalho, à baixa interação interprofissional e ao ambiente de trabalho precário.

Além disso, este artigo indica que estratégias de suporte devem ser utilizadas pela equipe de enfermagem oncológica para enfrentar os riscos ocupacionais e propiciar a manutenção da segurança laboral, entre elas: a educação continuada; o controle do ambiente de trabalho com recursos disponíveis; reforma da estrutura física e aquisição de mobiliário funcional e ergonômico; iluminação e ventilação adequadas dos setores; aumento do quantitativo de profissionais a fim de reduzir o ritmo e a demanda de trabalho; e suporte organizacional. Boa parte dos estudos, no entanto, recomendam o processo educativo por meio de treinamentos, cursos e palestras. Para tanto, o diagnóstico situacional é etapa fundamental para a implantação das intervenções, ressaltado como é importante que a instituição e a efetivação das estratégias garantam o engajamento de todas as pessoas envolvidas no processo de trabalho.

\section{Contribuições de autoria}

Fernandes MA, Rocha DM, Ribeiro HKP e Sousa CCM tiveram contribuição substancial na concepção do estudo, no levantamento, análise e interpretação dos dados, na elaboração e revisão crítica do manuscrito e na aprovação da versão final publicada, e assumem responsabilidade pública integral pelo trabalho realizado e pelo conteúdo publicado.

\section{Referências}

1. Silva SM, Baptista PCP, Felli VEA, Martins AC, Sarquis LMM, Mininel VA. Estratégias de intervenção relativas à saúde dos trabalhadores de enfermagem de hospitais universitários no Brasil. Rev Lat Am Enfermagem [Internet]. 2013 [citado em 3 mar 2018];21(1):[9 telas]. Disponível em: http:// www.scielo.br/pdf/rlae/v21n1/pt_v21n1a03.pdf

2. Loro MM, Zeitoune RCG. Collective strategy for facing occupational risks of a nursing team. Rev Esc Enferm USP [Internet]. 2017 [citado em 3 mar 2018];51:e03205. Disponível em: https://www.scielo.br/j/reeusp/a/ GwCwDbHB5sVbxWRn6h3bcHC/ ?lang =en

3. Silva SM, Telles ACM, Gallasch CH, Almeida MCS, Baptista PCP, Felli VEA. Temáticas investigadas pelo Grupo de Estudos sobre a Saúde do Trabalhador de Enfermagem e Saúde. Rev Enferm UERJ [Internet]. 2016 [citado em 3 mar 2018];24(5):e22974. Disponível em: http:// www.e-publicacoes.uerj.br/index.php/ enfermagemuerj/article/view/22974/20184
4. Miguel DB, Loro MM, Rosanelli CLSP, Kolankiewicz ACB, Stumm EMF, Zeitoune RCG. Percepção de trabalhadores de uma unidade oncológica acerca dos riscos ocupacionais. Cienc Cuid Saude [Internet]. 2014 [citado em 3 mar 2018];13(3):52734. Disponível em: https://periodicos.uem.br/ojs/ index.php/CiencCuidSaude/article/download/21208/ pdf_225/\#: :text=Os\%20riscos\%20apontados\% 20foram\%20inerentes,a\%20import\%C3\%A2ncia\% 20de\%20utiliz\%C3\%A1\%2Dlas

5. Umann J, Silva RM, Benavente SBT, Guido LA. O impacto das estratégias de enfrentamento na intensidade de estresse de enfermeiras de hematooncologia. Rev Gaucha Enferm [Internet]. 2014 [citado em 3 mar 2018];35(3):103-10. Disponível em: http://www.scielo.br/pdf/rgenf/v35n3/pt_19831447-rgenf-35-03-00103.pdf

6. Steel J, Godderis L, Luyten J. Productivity estimation in economic evaluations of occupational health and safety interventions: a systematic review. Scand J 
Work Environ Health [Internet]. 2018 [citado em 3 mar 2018];44(5):458-74. Disponível em: http://www. sjweh.fi/show_abstract.php?abstract_id=3715

7. MacEachen E, Kosny A, Ståhl C, O’Hagan F, Redgrift L, Sanford S, et al. Systematic review of qualitative literature on occupational health and safety legislation and regulatory enforcement planning and implementation. Scand J Work Environ Health [Internet]. 2016 [citado em 3 mar 2018];42(1):3-16. Disponível em: http:// www.sjweh.fi/show_abstract.php?abstract_ id $=3529 \&$ fullText $=\overline{1}$ \#box-fullText

8. Santos NAR, Santos J, Silva VR, Passos JP. Estresse ocupacional na assistência de cuidados paliativos em oncologia. Cogitare Enferm [Internet]. 2017 [citado em 3 mar 2018];22(4):e50686. Disponível em: https://revistas.ufpr.br/cogitare/article/view/ 50686/pdf

9. Donato H, Donato M. Etapas na condução de uma revisão sistemática. Acta Med Port [Internet]. 2019 [citado em 4 abr 2020];32(3):227-35. Disponível em: https://www.actamedicaportuguesa.com/ revista/index.php/amp/article/view/11923/5635

10. Santos CMC, Pimenta CAM, Nobre MRC. The PICO strategy for the research question construction and evidence search. Rev Lat Am Enfermagem [Internet]. 2007 [citado em 19 fev 2018];15(3):50811. Disponível em: http://www.scielo.br/scielo. php?script $=$ sci arttext\&pid $=$ S010411692007000300023

11. Crickman R, Finnell DS. Chemotherapy Safe Handling: Limiting Nursing Exposure With a Hazardous Drug Control Program. Clin J Oncol Nurs [Internet]. 2017 [citado em 10 mar 2018];21(1):73-8. Disponível em: https:/cjon.ons.org/cjon/21/1/ chemotherapy-safe-handling-limiting-nursingexposure-hazardous-drug-control-program

12. De la Fuente-Solana EI, Gómez-Urquiza JL, Cañadas GR, Albendín-García L, Ortega-Campos E, Cañadas-De la Fuente GA. Burnout and its relationship with personality factors in oncology nurses. Eur J Oncol Nurs [Internet]. 2017 [citado em 10 mar 2018];30:91-6. Disponível em: https:// www.ejoncologynursing.com/article/S14623889(17)30193-X/fulltext

13. Colvin CM, Karius D, Albert NM. Nurse Adherence to Safe-Handling Practices: Observation Versus Self-Assessment. Clin J Oncol Nurs [Internet]. 2016 [citado em 10 mar 2018];20(6):617-22. Disponível em: https://cjon.ons.org/cjon/20/6/nurse-adherencesafe-handling-practices-observation-versus-selfassessment

14. Karanikola MNK, Giannakopoulou M, Kalafati M, Kaite CP, Patiraki E, Mpouzika M, et al. Anxiety symptoms and quality of interaction among oncology nurses: a correlational, cross-sectional study. Rev Esc Enferm USP [Internet]. 2016 [citado em 10 mar 2018];50(5):800-7. Disponível em: https:/www.scielo.br/j/reeusp/a/PDk3Y88HD8nMFt $\mathrm{SJ} 3 \mathrm{bbLLvN} /$ ?format $=$ pdf\&lang $=\mathrm{en}$
15. Friese CR, Mendelsohn-Victor K, Wen B, Sun D, Sutcliffe K, Yang JJ, et al. DEFENS - Drug Exposure Feedback and Education for Nurses' Safety: study protocol for a randomized controlled trial. Trials [Internet]. 2015 [citado em 10 mar 2018];16:171. Disponível em: https://trialsjournal.biomedcentral. com/articles/10.1186/s13063-015-0674-5

16. Davidson JE, Agan DL, Chakedis S. Exploring Distress Caused by Blame for a Negative Patient Outcome. J Nurs Adm [Internet]. 2016 [citado em 10 mar 2018];46(1):18-24. Disponível em: https:// journals.lww.com/jonajournal/Abstract/2016/01000/ Exploring_Distress_Caused_by_Blame_for_a_ Negative.6.aspx

17. Trindade LL, Bordignon M, Ferraz L, Amestoy SC. Professional satisfaction and quality of care in oncology: the vision of health professionals. Rev Pesqui (Univ Fed Estado Rio J) [Internet]. 2015 [citado em 10 mar 2018];7(2):2383-92. Disponível em: http://www.seer.unirio.br/index.php/ cuidadofundamental/article/view/3895/pdf_1550

18. Poulsen AA, Sharpley CF, Baumann KC, Henderson J, Poulsen MG. Evaluation of the effect of a 1-day interventional workshop on recovery from job stress for radiation therapists and oncology nurses: A randomised trial. J Med Imaging Radiat Oncol [Internet]. 2015 [citado em 10 mar 2018];59(4):491-8. Disponível em: https:/onlinelibrary.wiley.com/ doi/10.1111/1754-9485.12322

19. Nascimento L, Loro MM, Stumm EMF, Kirchner RM, Rosanelli CLSP, Kolankiewicz ACB. Occupational risks of nursing work in an oncology unit. Rev Enferm UFPE On Line [Internet]. 2011 [citado em 10 mar 2018];5(6):1403-10. Disponível em: https://periodicos.ufpe.br/revistas/ revistaenfermagem/article/viewFile/6809/6057

20. Ko W, Kiser-Larson N. Stress Levels of Nurses in Oncology Outpatient Units. Clin J Oncol Nurs [Internet]. 2016 [citado em 3 mar 2018];20(2):158-64. Disponível em: https:/cjon.ons.org/cjon/20/2/ stress-levels-nurses-oncology-outpatient-units

21. Chen HC, Lu ZY, Lee SH. Nurses' Experiences in Safe Handling of Chemotherapeutic Agents: The Taiwan Case. Cancer Nurs [Internet]. 2016 [citado em 10 mar 2018];39(5):e29-38. Disponível em: https://journals.lww.com/cancernursingonline/ Abstract/2016/09000/Nurses_Experiences_in_ Safe_Handling_of.14.aspx

22. Jeong KW, Lee BY, Kwon MS, Jang JH. Safety Management Status among Nurses Handling Anticancer Drugs: Nurse Awareness and Performance Following Safety Regulations. Asian Pac J Cancer Prev [Internet]. 2015 [citado em 10 mar 2018];16(8):3203-11. Disponível em: http://koreascience.or.kr/ article/JAKO201528551641014.page

23. Umann J, Silva RM, Benetti ERR, Guido LA. Stress and coping among nurses of hemato-oncologic units. Rev Rene [Internet]. 2013 [citado em 10 mar 2018];14(4):783-90. Disponível em: http://www. periodicos.ufc.br/rene/article/view/3546/2786 
24. Polovich M, Clark PC. Factors influencing oncology nurses' use of hazardous drug safe-handling precautions. Oncol Nurs Forum [Internet]. 2012 [citado em 10 mar 10 2018];39(3):e299-309. Disponível em: https:/onf.ons.org/onf/39/3/factorsinfluencing-oncology-nurses-use-hazardous-drugsafe-handling-precautions

25. Polovich M, Martin S. Nurses' use of hazardous drug-handling precautions and awareness of national safety guidelines. Oncol Nurs Forum [Internet]. 2011 [citado em 10 mar 2018];38(6):718-26. Disponível em: https://onf.ons.org/onf/38/6/nurses-use-hazardousdrug-handling-precautions-and-awareness-nationalsafety-guidelines

26. Baykal U, Seren S, Sokmen S. A description of oncology nurses' working conditions in Turkey. Eur J Oncol Nurs [Internet]. 2009 [citado em 10 mar 2018];13(5):368-75. Disponível em: https:// www.ejoncologynursing.com/article/S14623889(09)00056-8/fulltext

27. Rodrigues AB, Chaves EC. Fatores estressantes e estratégias de coping dos enfermeiros atuantes em oncologia. Rev Lat Am Enfermagem [Internet]. 2008 [citado em 10 mar 2018];16(1):[5p]. Disponível em: https://www.scielo.br/j/rlae/a/
m9Kgw8zXQ8bcWPHLFNmdThh/?lang=pt\& format $=\mathrm{pdf}$

28. Santos NAR, Gomes SV, Rodrigues CMA, Santos J, Passos JP. Estratégias de enfrentamento utilizadas pelos enfermeiros em cuidados paliativos oncológicos: revisão integrativa. Cogitare Enferm [Internet]. 2016 [citado em 10 jun 2019];21(3):1-8. Disponível em: http://www.redalyc.org/ jatsRepo/4836/483653826025/483653826025.pdf

29. Maurício LFS, Okuno MFP, Campanharo CRV, Lopes MCBT, Belasco AGS, Batista REA. Professional nursing practice in critical units: assessment of work environment characteristics. Rev Lat Am Enfermagem [Internet]. 2017 [citado em 10 mar 2018];25:e2854. Disponível em: https:// www.scielo.br/j/rlae/a/sz8CcKdfkDFgDZmjfbqwSg $\mathrm{R} /$ ?lang $=\mathrm{en}$

30. Nazario EG, Camponogara S, Dias GL. Occupational risks and adherence to standard precautions in intensive care nursing work: workers' perceptions. Rev Bras Saude Ocup [Internet]. 2017 [citado em 10 mar 2018];42:e7. Disponível em: http://www.scielo.br/pdf/rbso/v42/ en_2317-6369-rbso-42-e7.pdf 